\title{
Staged self-assembly: nanomanufacture of arbitrary shapes with $O(1)$ glues
}

\author{
Erik D. Demaine - Martin L. Demaine - Sándor P. Fekete • \\ Mashhood Ishaque $\cdot$ Eynat Rafalin $\cdot$ Robert T. Schweller • \\ Diane L. Souvaine
}

\begin{abstract}
We introduce staged self-assembly of Wang tiles, where tiles can be added dynamically in sequence and where intermediate constructions can be stored for later mixing. This model and its various constraints and performance measures are motivated by a practical nanofabrication scenario through protein-based bioengineering. Staging allows us to break through the traditional lower bounds in tile self-assembly by encoding the shape in the staging algorithm instead of the tiles. All of our results are based on the
\end{abstract}

E. D. Demaine · M. L. Demaine

MIT Computer Science and Artificial Intelligence Laboratory, 32 Vassar St., Cambridge, MA 02139, USA

e-mail: edemaine@mit.edu

M. L. Demaine

e-mail: mdemaine@mit.edu

S. P. Fekete

Department of Computer Science, Braunschweig University of Technology,

Muehlenpfordtstr. 23, Braunschweig 38116, Germany

e-mail: s.fekete@tu-bs.de

M. Ishaque - D. L. Souvaine

Department of Computer Science, Tufts University,

Medford, MA 02155, USA

e-mail: mishaq01@cs.tufts.edu

D. L. Souvaine

e-mail: dls@cs.tufts.edu

E. Rafalin

Google Inc., Mountain View, CA 94043, USA

e-mail: erafalin@cs.tufts.edu

R. T. Schweller ( $\square)$

Department of Computer Science, University of Texas-Pan American,

Edinburg, TX 78539, USA

e-mail: schwellerr@cs.panam.edu 
practical assumption that only a constant number of glues, and thus only a constant number of tiles, can be engineered. Under this assumption, traditional tile self-assembly cannot even manufacture an $n \times n$ square; in contrast, we show how staged assembly in theory enables manufacture of arbitrary shapes in a variety of precise formulations of the model.

Keywords Self-assembly $\cdot$ Tiling $\cdot$ Nanotechnology $\cdot$ DNA computing · DNA self-assembly

\section{Introduction}

Self-assembly is the process by which an organized structure can form spontaneously from simple parts. It describes the assembly of diverse natural structures such as crystals, DNA helices, and microtubules. In nanofabrication, the idea is to co-opt natural self-assembly processes to build desired structures, such as a sieve for removing viruses from serum, a drugdelivery device for targeted chemotherapy or brachytherapy, a magnetic device for medical imaging, a catalyst for enzymatic reactions, or a biological computer. Self-assembly of artificial structures has promising applications to nanofabrication and biological computing. The general goal is to design and manufacture nanoscale pieces (e.g., strands of DNA) that self-assemble uniquely into a desired macroscale object (e.g., a computer).

Our work is motivated and guided by an ongoing collaboration with the Sackler School of Graduate Biomedical Sciences that aims to nanomanufacture sieves, catalysts, and drugdelivery and medical-imaging devices, using protein self-assembly. Specifically, the Goldberg Laboratory is currently developing technology to bioengineer (many copies of) rigid struts of varying lengths, made of several proteins, which can join collinearly to each other at compatible ends. These struts occur naturally as the "legs" of the T4 bacteriophage, a virus that infects bacteria by injecting DNA. In contrast to nanoscale selfassembly based on DNA (Winfree et al. 1998; Mao et al. 2000; Rothemund et al. 2004; Barish et al. 2005; Seeman 1998; Shih et al. 2004; Rothemund 2006), which is inherently floppy, these nanorod structures are extremely rigid and should therefore scale up to the manufacture of macroscale objects.

The traditional, leading theoretical model for self-assembly is the two-dimensional tile assembly model introduced by Winfree in his Ph.d. thesis (Winfree 1998) and first appearing at STOC 2000 (Rothemund and Winfree 2000). The basic building blocks in this model are Wang tiles (Wang 1961), unrotatable square tiles with a specified glue on each side, where equal glues have affinity and may stick. Tiles then self-assemble into supertiles: two (super)tiles nondeterministically join if the sum of the glue affinities along the attachment is at least some threshold $\tau$, called temperature. This basic model has been generalized and extended in many ways (Adleman 2000; Adleman et al. 2001, 2002; Soloveichik and Winfree 2004; Aggarwal et al. 2005; Rothemund and Winfree 2000; Kao and Schweller 2006). The model should be practical because Wang tiles can easily simulate the practical scenario in which tiles are allowed to rotate, glues come in pairs, and glues have affinity only for their unique mates. In particular, we can implement such tiles using two unit-length nanorods joined at right angles at their midpoints to form a plus sign.

Most theoretical research in self-assembly considers the minimum number of distinct tiles - the tile complexity $t$-required to assemble a shape uniquely. In particular, if we allow the desired shape to be scaled by a possibly very large factor, then in most models the minimum possible tile complexity (the smallest "tile program") is $\Theta(K / \lg K)$ where $K$ is the Kolmogorov complexity of the shape (Soloveichik and Winfree 2004). In practice, 
the limiting factor is the number of distinct glues-the glue complexity g-as each new glue type requires significant biochemical research and experiments. For example, a set of DNA-based glues requires experiments to test whether a collection of codewords have a "conflict" (a pair of noncomplementary base sequences that attach to each other), while a set of protein-based glues requires finding pairs of proteins with compatible geometries and amino-acid placements that bind (and no other pairs of which accidentally bind). Of course, tile and glue complexities are related: $g \leq t \leq g^{4}$.

We present the staged tile assembly model, a generalization of the tile assembly model that captures the temporal aspect of the laboratory experiment, and enables substantially more flexibility in the design and fabrication of complex shapes using a small tile and glue complexity. In its simplest form, staged assembly enables the gradual addition of specific tiles in a sequence of stages. In addition, any tiles that have not yet attached as part of a supertile can be washed away and removed (in practice, using a weight-based filter, for example). More generally, we can have any number of bins (in reality, batches of liquid solution stored in separate containers), each containing tiles and/or supertiles that self-assemble as in the standard tile assembly model. During a stage, we can perform any collection of operations of two types: (1) add (arbitrarily many copies of) a new tile to an existing bin; and (2) pour one bin into another bin, mixing the contents of the former bin into the latter bin, and keeping the former bin intact. In both cases, any pieces that do not assemble into larger structures are washed away and removed. These operations let us build intermediate supertiles in isolation and then combine different supertiles as whole structures. Now we have two new complexity measures in addition to tile and glue complexity: the number of stages-or stage complexity $s$-measures the time required by the operator of the experiment, while the number of bins-or bin complexity $b$-measures the space required for the experiment. ${ }^{1}$ (When both of these complexities are 1, we obtain the regular tile assembly model.)

Our results. We show that staged assembly enables substantially more efficient manufacture in terms of tile and glue complexity, without sacrificing much in stage and bin complexity. All of our results assume the practical constraint of having only a small constant number of glues and hence a constant number of tiles. In contrast, an informationtheoretic argument shows that this assumption would limit the traditional tile assembly model to constructing shapes of constant Kolmogorov complexity.

For example, we develop a method for self-assembling an $n \times n$ square for arbitrary $n>0$, using 16 glues and thus $O(1)$ tiles (independent of $n)$, and using only $O(\log \log n)$ stages, $O(\sqrt{\log n})$ bins, and temperature $\tau=2$ (Sect. 4.2). Alternatively, with the minimum possible temperature $\tau=1$, we can self-assemble an $n \times n$ square using 9 glues, $O(1)$ tiles and bins, and $O(\log n)$ stages (Sect. 4.1). In contrast, the best possible self-assembly of an $n \times n$ square in the traditional tile assembly model has tile complexity $\Theta(\log n / \log \log n)$ (Adleman et al. 2001; Rothemund and Winfree 2000), or $\Theta(\sqrt{\log n})$ in a rather extreme generalization of allowable pairwise glue affinities (Aggarwal et al. 2005).

More generally, we show how to self-assemble arbitrary shapes made up of $n$ unit squares in a variety of precise formulations of the problem. Our simplest construction builds the shape using 2 glues, 16 tiles, $O$ (diameter) stages, and $O(1)$ bins, but it only glues tiles together according to a spanning tree, which is what we call the partial connectivity model (Sect. 5.1). All other constructions have full connectivity: any two adjacent unit

\footnotetext{
${ }^{1}$ Here we view the mixing time required in each stage (and the volume of each bin) as a constant, mainly because it is difficult to analyze precisely from a thermodynamic perspective, as pointed out in (Adleman 2000). In our constructions, we believe that a suitable design of the relative concentrations of tiles (a feature not captured by the model) leads to reasonable mixing times.
} 
squares are built by tiles with matching glues along their shared edge. In particular, if we scale an arbitrary hole-free shape larger by a factor of 2 , then we can self-assemble with full connectivity using 8 glues, $O(1)$ tiles, and $O(n)$ stages and bins (Sect. 5.2). We also show how to simulate a traditional tile assembly construction with $t$ tiles by a staged assembly using 3 glues, $O(1)$ tiles, $O(\log \log t)$ stages, $O(t)$ bins, and a scale factor of $O(\log t)($ Sect. 5.3). If the shape happens to be monotone in one direction, then we can avoid scaling and still obtain full connectivity, using 9 glues, $O(1)$ tiles, $O(\log n)$ stages, and $O(n)$ bins (Sect. 5.4). We also discuss an efficient method for the design of binary counters in the staged assembly framework, an important tool for a large number of selfassembly systems (Sect. 6). This technique offers benefits over non-staged counters in terms of reduced temperature $(\tau=1)$ and potentially faster assembly.

Table 1 summarizes our results in more detail, in particular elaborating on possible tradeoffs between the complexities. The table captures one additional aspect of our constructions: Planarity. Consider two jigsaw puzzle pieces with complex borders lying on a flat surface. It may not be possible to slide the two pieces together while both remain on the table. Rather, one piece must be lifted off the table and dropped into position. Our current model of assembly intuitively permits supertiles to be placed into position from the third dimension, despite the fact that it may not be possible to assemble within the plane. A planar construction guarantees assembly of the final target shape even if we restrict assembly of supertiles to remain completely within the plane. This feature seems desirable, though it may not be essential in two dimensions because reality will always have some thickness in the third dimension (2.5D). However, the planarity constraint (or spatiality constraint in 3D) becomes more crucial in 3D assemblies, where there is no fourth dimension to avoid intersection, so this feature gives an indication of which methods might generalize to 3D; see Sect. 7.

Related work. There are a handful of existing works in the field of DNA self-assembly that have proposed very basic multiple stage assembly procedures. John Reif (1999)

Table 1 Summary of the glue, tile, bin, and stage complexities, the temperature $\tau$, the scale factor, the connectivity, and the planarity of our staged assemblies and the relevant previous work

\begin{tabular}{|c|c|c|c|c|c|c|c|c|}
\hline & Glues & Tiles & Bins & Stages & $\tau$ & Scale & Conn. & Planar \\
\hline \multicolumn{9}{|l|}{$n \times n$ square } \\
\hline $\begin{array}{l}\text { Previous work (Adleman } \\
\text { et al. 2001; Rothemund } \\
\text { and Winfree 2000) }\end{array}$ & \multicolumn{2}{|c|}{$\Theta\left(\frac{\log n}{\log \log n}\right)$} & 1 & 1 & 2 & 1 & Full & Yes \\
\hline Jigsaw technique (Sect. 4.2) & 9 & $O(1)$ & $O(1)$ & $O(\log n)$ & 1 & 1 & Full & Yes \\
\hline Crazy mixing (Sect. 4.2) & 16 & $O(1)$ & $B$ & $O\left(\left\lceil\frac{\log n}{B^{2}}\right\rceil+\log B\right)$ & 2 & 1 & Full & Yes \\
\hline Crazy mixing, $B=\sqrt{\log n}$ & 16 & $O(1)$ & $\sqrt{\log n}$ & $O(\log \log n)$ & 2 & 1 & Full & Yes \\
\hline \multicolumn{9}{|l|}{ General shape with $n$ tiles } \\
\hline $\begin{array}{l}\text { Previous work (Soloveichik } \\
\text { and Winfree 2004) }\end{array}$ & \multicolumn{2}{|c|}{$\Theta(K / \log K)$} & 1 & 1 & 2 & Unbounded & Partial & No \\
\hline $\begin{array}{l}\text { Arbitrary shape with } n \text { tiles } \\
\quad \text { (Sect. 5.1) }\end{array}$ & 2 & 16 & $O(\log n)$ & $O$ (diameter) & 1 & 1 & Partial & No \\
\hline $\begin{array}{l}\text { Hole-free shape with } n \text { tiles } \\
\quad \text { (Sect. 5.2) }\end{array}$ & 8 & $O(1)$ & $O(n)$ & $O(n)$ & 1 & 2 & Full & No \\
\hline $\begin{array}{l}\text { Simulation of 1-stage tiles } T \\
\quad \text { (Sect. 5.3) }\end{array}$ & 3 & $O(1)$ & $O(|T|)$ & $O(\log \log |T|)$ & 1 & $O(\log |T|)$ & Partial & No \\
\hline $\begin{array}{l}\text { Monotone shapes with } n \text { tiles } \\
\text { (Sect. 5.4) }\end{array}$ & 9 & $O(1)$ & $O(n)$ & $O(\log n)$ & 1 & 1 & Full & Yes \\
\hline
\end{tabular}


introduced a step-wise assembly model for local parallel biomolecular computing. In more recent work Park et al. (2006) have considered a simple hierarchical assembly technique for the assembly of DNA lattices. Somei et al. (2006) have considered a microfluidic device for stepwise assembly of DNA tiles. While all of these works use some form of stepwise or staged assembly, they do not study the complexity of staged assembly to the depth that we do here. Further, none consider the concept of bin complexity.

\section{The staged assembly model}

In this section, we present basic definitions common to most assembly models, then we describe the staged assembly model, and finally we define various metrics to measure the efficiency of a staged assembly system.

Tiles and tile systems. A (Wang) tile $t$ is a unit square defined by the ordered quadruple $\langle\operatorname{north}(t), \operatorname{east}(t), \operatorname{south}(t)$, west $(t)\rangle$ of glues on the four edges of the tile. Each glue is taken from a finite alphabet $\Sigma$, which includes a special "null" glue denoted null. For simplicity of bounds, we do not count the null glue in the glue complexity $g=|\Sigma|-1$.

A tile system is an ordered triple $\langle T, G, \tau\rangle$ consisting of the tileset $T$ (a set of distinct tiles), the glue function $G: \Sigma^{2} \rightarrow\{0,1, \ldots, \tau\}$, and the temperature $\tau$ (a positive integer). It is assumed that $G(x, y)=G(y, x)$ for all $x, y \in \Sigma$ and that $G($ null, $x)=0$ for all $x \in \Sigma$. Indeed, in all of our constructions, as in the original model of Adleman (2000), $G(x, y)=0$ for all $x \neq y$ (see footnote ${ }^{2}$ ), and each $G(x, x) \in\{1,2, \ldots, \tau\}$. The tile complexity of the system is $|T|$.

Configurations. Define a configuration to be a function $C: \mathbb{Z}^{2} \rightarrow T \cup\{$ empty $\}$, where empty is a special tile that has the null glue on each of its four edges. The shape of a configuration $C$ is the set of positions $(i, j)$ that do not map to the empty tile. The shape of a configuration can be disconnected, corresponding to several distinct supertiles.

Adjacency graph and supertiles. Define the adjacency graph $G_{C}$ of a configuration $C$ as follows. The vertices are coordinates $(i, j)$ such that $C(i, j) \neq$ empty. There is an edge between two vertices $\left(x_{1}, y_{1}\right)$ and $\left(x_{2}, y_{2}\right)$ if and only if $\left|x_{1}-x_{2}\right|+\left|y_{1}-y_{2}\right|=1$. A supertile is a maximal connected subset $G^{\prime}$ of $G_{C}$, i.e., $G^{\prime} \subseteq G_{C}$ such that, for every connected subset $H$, if $G^{\prime} \subseteq H \subseteq G_{C}$, then $H=G^{\prime}$. For a supertile $S$, let $|S|$ denote the number of nonempty positions (tiles) in the supertile. Throughout this paper, we will informally refer to (lone) tiles as a special case of supertiles.

If every two adjacent tiles in a supertile share a positive strength glue type on abutting edges, the supertile is fully connected.

Two-handed assembly and bins. Informally, in the two-handed assembly model, any two supertiles may come together (without rotation or flipping) and attach if their strength of attachment, from the glue function, meets or exceeds a given temperature parameter $\tau$.

Formally, for any two supertiles $X$ and $Y$, the combination set $C_{(X, Y)}^{\tau}$ of $X$ and $Y$ is defined to be the set of all supertiles obtainable by placing $X$ and $Y$ adjacent to each other (without overlapping) such that, if we list each newly coincident edge $e_{i}$ with edge strength $s_{i}$, then $\sum s_{i} \geq \tau$.

We define the assembly process in terms of bins. Intuitively, a bin consists of an initial collection of supertiles that self-assemble at temperature $\tau$ to produce a new set of supertiles $P$. Formally, with respect to a given set of tile-types $T$, a $\operatorname{bin}$ is a pair $(S, \tau)$ where $S$

\footnotetext{
${ }^{2}$ With a typical implementation in DNA, glues actually attach to unique complements rather than to themselves. However, this depiction of the glue function is standard in the literature and does not affect the power of the model.
} 
is a set of initial supertiles whose tile-types are contained in $T$, and $\tau$ is a temperature parameter. For a bin $(S, \tau)$, the set of produced supertiles $P_{(S, \tau)}^{\prime}$ is defined recursively as follows: (1) $S \subseteq P_{(S, \tau)}^{\prime}$ and (2) for any $X, Y \in P_{(S, \tau)}^{\prime}, C_{(X, Y)}^{\tau} \subseteq P_{P}^{\prime}{ }_{(S, \tau)}$. The set of terminally produced supertiles of a bin $(S, \tau)$ is $P_{(S, \tau)}=\left\{X \in P^{\prime} \mid Y \in P^{\prime}, C_{(X, Y)}^{\tau}=\emptyset\right\}$. We say the set of supertiles $P$ is uniquely produced by bin $(S, \tau)$ if each supertile in $P^{\prime}$ is of finite size. Put another way, unique production implies that every producible supertile can grow into a supertile in $P$.

Intuitively, $P^{\prime}$ represents the set of all possible supertiles that can self-assemble from the initial set $S$, whereas $P$ represents only the set of supertiles that cannot grow any further. In the case of unique assembly of $P$, the latter thus represents the eventual, final state of the self-assembly bin. Our goal is therefore to produce bins that yield desired supertiles in the uniquely produced set $P$.

Given a collection of bins, we model the process of mixing bins together in arbitrarily specified patterns in a sequence of distinct stages. In particular, we permit the following actions: We can create a bin of a single tile type $t \in T$, we can merge multiple bins together into a single bin, and we can split the contents of a given bin into multiple new bins. In particular, when splitting the contents of a bin, we assume the ability to extract only the unique terminally produced set of supertiles $P$, while filtering out additional partial assemblies in $P^{\prime}$. Intuitively, given enough time for assembly and a large enough volume of tiles, a bin that uniquely produces $P$ should consist of almost entirely the terminally produced set $P$. We formally model the concept of mixing bins in a sequence of stages with the mix graph.

Mix graphs. An $r$-stage b-bin mix graph $M$ consists of $r b+1$ vertices, $m_{*}$ and $m_{i, j}$ for $1 \leq i \leq r$ and $1 \leq j \leq b$, and an arbitrary collection of edges of the form $\left(m_{r, j}, m_{*}\right)$ or $\left(m_{i, j}, m_{i+1, k}\right)$ for some $i, j, k$.

Staged assembly systems. A staged assembly system is a 3-tuple $\left\langle M_{r, b},\left\{T_{i, j}\right\},\left\{\tau_{i, j}\right\}\right\rangle$ where $M_{r, b}$ is an $r$-stage $b$-bin mix graph, each $T_{i, j}$ is a set of tile types, and each $\tau_{i, j}$ is an integer temperature parameter. Given a staged assembly system, for each $1 \leq i \leq r, 1 \leq j \leq b$, we define a corresponding bin $\left(R_{i, j}, \tau_{i, j}\right)$ where $R_{i, j}$ is defined as follows:

1. $R_{1, j}=T_{1, j}$ (this is a bin in the first stage);

2. For $i \geq 2, R_{i, j}=\left(\bigcup_{k:\left(m_{i-1, k}, m_{i, j}\right) \in M_{r, b}} P_{\left(R_{(i-1, k)}, \tau_{i-1, k}\right)}\right) \cup T_{i, j}$.

3. $\quad R_{*}=\bigcup_{k:\left(m_{r, k}, m_{*}\right) \in M_{r, b}} P_{\left(R_{(r, k)}, \tau_{r, k)}\right)}$.

Thus, the $j$ th bin in the $i$ th stage takes its initial set of seed supertiles to be the terminally produced supertiles from a collection of bins from the previous stage, the exact collection specified by $M_{r, b}$, in addition to a set of added tile types $T_{i, j}$. Intuitively, the mix graph specifies how each collection of bins should be mixed together when transitioning from one stage to the next. We define the set of terminally produced supertiles for a staged assembly system to be $P_{\left(R_{*}, \tau_{*}\right)}$. In this paper, we are interested in staged assembly systems for which each bin yields unique assembly of terminal supertiles. In this case we say a staged assembly system uniquely produces the set of supertiles $P_{\left(R_{*}, \tau_{*}\right)}$.

Throughout this paper, we assume that, for all $i, j, \tau_{i, j}=\tau$ for some fixed global temperature $\tau$, and we denote a staged assembly system as $\left\langle M_{r, b},\left\{T_{i, j}\right\}, \tau\right\rangle$.

Metrics. We are interested in designing efficient staged assembly systems that terminally produce a unique target shape. We use the following natural metrics to measure the efficiency of the staged tile system: 
Tile types:

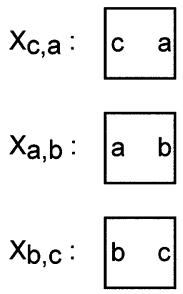

Tile Sets:

$T_{1,1}=\left\{x_{a, b}, x_{b, c}\right\}$

$T_{1,2}=\left\{x_{a, b}, x_{c, a}\right\}$

$\mathrm{T}_{2,1}=\{\}$

$\mathrm{T}_{2,2}=\{\}$

$\mathrm{T}_{3,1}=\left\{\mathrm{x}_{\mathrm{b}, \mathrm{c}}\right\}$

$\mathrm{T}_{3,2}=\left\{\mathrm{x}_{\mathrm{c}, \mathrm{a}}\right\}$
Mix Graph:

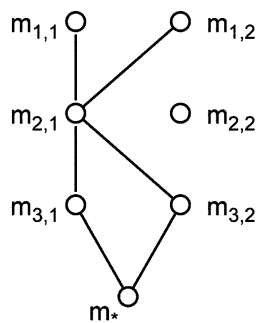

Uniquely produced supertile:

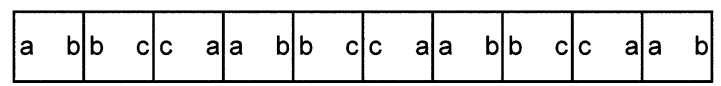

Fig. 1 A sample staged assembly system that uniquely assembles a $1 \times 10$ line. The temperature is $\tau=1$, and each glue $a, b, c$ has strength 1 . The tile, stage, and bin complexities are 3,3 , and 2 , respectively

Tile complexity: $\left|\bigcup T_{i, j}\right|$. This represents the number of distinct tile types that the assembly system requires. In this paper we emphasize $O(1)$ tile complexity systems in contrast to previous work, see Fig. 1 for an example staged assembly system.

Bin complexity: The number $b$ of vertices in each partition of the mix graph. Intuitively this measures the number of distinct containers that would be required to carry out the specified staged assembly procedure.

Stage complexity: The number $r$ of sequential stages of mixing that occur. This metric measures the number of stages in which collections of bins must be brought to their terminal assemblies and mixed together into a new array of bins. It represents operator time.

Temperature: The value $\tau$. In practice, it is difficult to implement systems with accurate temperature sensitivity. In this paper we focus on $\tau \in\{1,2,3\}$.

We also consider the following features to measure the quality of the shape produced:

Planarity: In a planar construction, supertiles have obstacle-free paths to reach their mates (Fig. 2).

Connectivity: In a fully connected supertile, every two adjacent tiles have the same positive-strength glue along their common edge. Otherwise the supertile is partially connected.

Scale factor: In some cases, we allow the produced shape to be a uniform scaling of the desired shape by some small positive integer, called the scale factor.

\section{Assembly of $1 \times n$ lines}

As a warmup, we develop a staged assembly for the $1 \times n$ rectangle ("line") using only three glues and $O(\log n)$ stages.

The assembly uses a divide-and-conquer approach to split the shape into a constant number of recursive pieces. Before we turn to the simple divide-and-conquer required here, we describe the general case, which will be useful later. This approach requires the pieces to be combinable in a unique way, forcing the creation of the desired shape. We consider the decomposition tree formed by the recursion, where sibling nodes should uniquely 
(a)

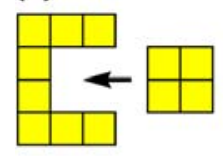

(b)

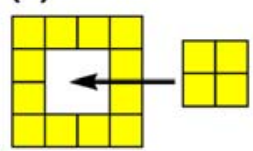

(c)

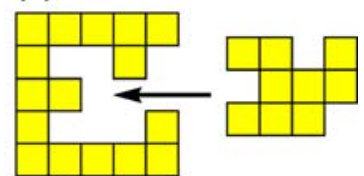

Fig. 2 All three assemblies are permitted under the basic model. However, only assembly (a) is permitted under the planarity constraint

assemble to their parent. The staging proceeds bottom-up in this tree. The height of this tree corresponds to the stage complexity, and the maximum number of distinct nodes at any level corresponds to the bin complexity. The idea is to assign glues to the pieces in the decomposition tree to guarantee unique assemblage while using few glues.

We now turn to constructing $1 \times 2^{k}$ lines:

Theorem 1 There is a planar temperature-1 staged assembly system that uniquely produces a (fully connected) $1 \times 2^{k}$ line using 3 glues, 6 tiles, 6 bins, and $O(k)$ stages.

Proof The decomposition tree simply splits a $1 \times 2^{k}$ line into two $1 n b s p ; \times n b s p ; 2^{k-1}$ lines. All tiles have the null glue on their top and bottom edges. If the $1 \times 2^{k}$ line has glue $a$ on its left edge, and glue $b$ on its right edge, then the left and right $1 \times 2^{k-1}$ inherit these glues on their left and right edges, respectively. We label the remaining two inner edgesthe right edge of the left piece and the left edge of the right piece-with a third glue $c$, distinct from $a$ and $b$. Because $a \neq b$, the left and right piece uniquely attach at the inner edges with common glue $c$. This recursion also maintains the invariant that $a \neq b$, so three glues suffice overall. Thus there are only $\left(\begin{array}{l}3 \\ 2\end{array}\right)=6$ possible $1 \times 2^{k}$ lines of interest, and we only need to store these six at any time, using six bins. At the base case of $k=0$, we just create the six possible single tiles. The number of stages beyond that creation is exactly $k$ (Fig. 3).

Corollary 1 There is a planar temperature-1 staged assembly system that uniquely produces a (fully connected) $1 \times n$ line using 3 glues, 6 tiles, 7 bins, and $O(\log n)$ stages.

Proof We augment the construction of Theorem 1 applied to $k=\lfloor\log n\rfloor$. When we build the $1 \times 2^{i}$ lines for some $i$, if the binary representation of $n$ has a 1 bit in the $i$ th position, then we add that line to a new output bin. Thus, in the output bin, we accumulate powers of 2 that sum to $n$. As in the proof of Theorem 1, three glues suffice to guarantee unique assemblage in the output bin. The number of stages remains $O(\log n)$.

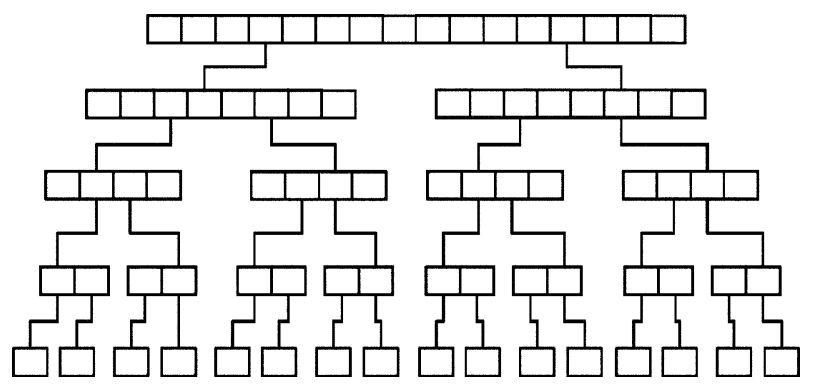

Fig. 3 Decomposition tree for $1 \times 16$ line 
(a)

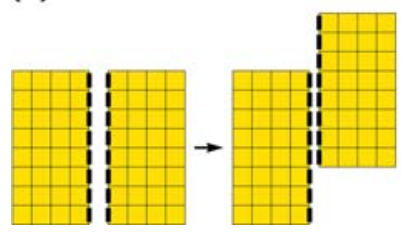

(b)

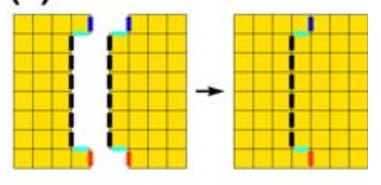

Fig. 4 (a) The shifting problem encountered when combining rectangle supertiles. (b) The jigsaw solution: two supertiles that combine uniquely into a fully connected square supertile

\section{Assembly of $n \times n$ squares}

Figure 4(a) illustrates the challenge with generalizing the decomposition-tree technique from $1 \times n$ lines to $n \times n$ squares. Namely, the naïve decomposition of a square into two $n \times n / 2$ rectangles cannot lead to a unique assembly using $O(1)$ glues with temperature 1 and full connectivity: by the pigeon-hole principle, some glue must be used more than once along the shared side of length $n$, and the lower part of the left piece may glue to the higher part of the right piece. Even though this incorrect alignment may make two unequal glues adjacent, in the temperature-1 model, a single matching pair of glues is enough for a possible assembly.

\subsection{Jigsaw technique}

To overcome this shifting problem, we introduce the jigsaw technique, a powerful tool used throughout this paper. This technique ensures that the two supertiles glue together uniquely based on geometry instead of glues. Figure 4(b) shows how to cut a square supertile into two supertiles with three different glues that force unique combination while preserving full connectivity.

Theorem 2 There is a planar temperature-1 staged assembly of a fully connected $n \times n$ square using 9 glues, $O(1)$ tiles, $O(1)$ bins, and $O(\log n)$ stages.

Proof We build a decomposition tree by first decomposing the $n \times n$ square by vertical cuts, until we obtain tall, thin supertiles; then we similarly decompose these tall, thin supertiles by horizontal cuts, until we obtain constant-size supertiles. Table 2 describes the general algorithm. Figure 5 shows the decomposition tree for an $8 \times 8$ square. The height of the decomposition tree, and hence the stage complexity, is $O(\log n)$.

We assign glue types to the boundaries of the supertiles to guarantee unique assemblage based on the jigsaw technique. The assignment algorithm is similar to the $1 \times n$ line, but we use three glues for the boundary of each supertile instead of one, for a total of nine glues instead of three. Figure 6 shows the glue assignment during the first two vertical decompositions of the $8 \times 8$ square.

It remains to show that the bin complexity is $O(1)$. We start by considering the vertical decomposition. At each level of the decomposition tree, there are three types of intermediate products: leftmost supertile, rightmost supertile and middle supertiles. The leftmost and rightmost supertiles are always in different bins. The important thing to observe is that the middle supertiles always have the same shape, though it is possible to have two different sizes - the number of columns can differ by one. In one of these sizes, the number of columns is even and, in the other, the number is odd. Thus we need separate 


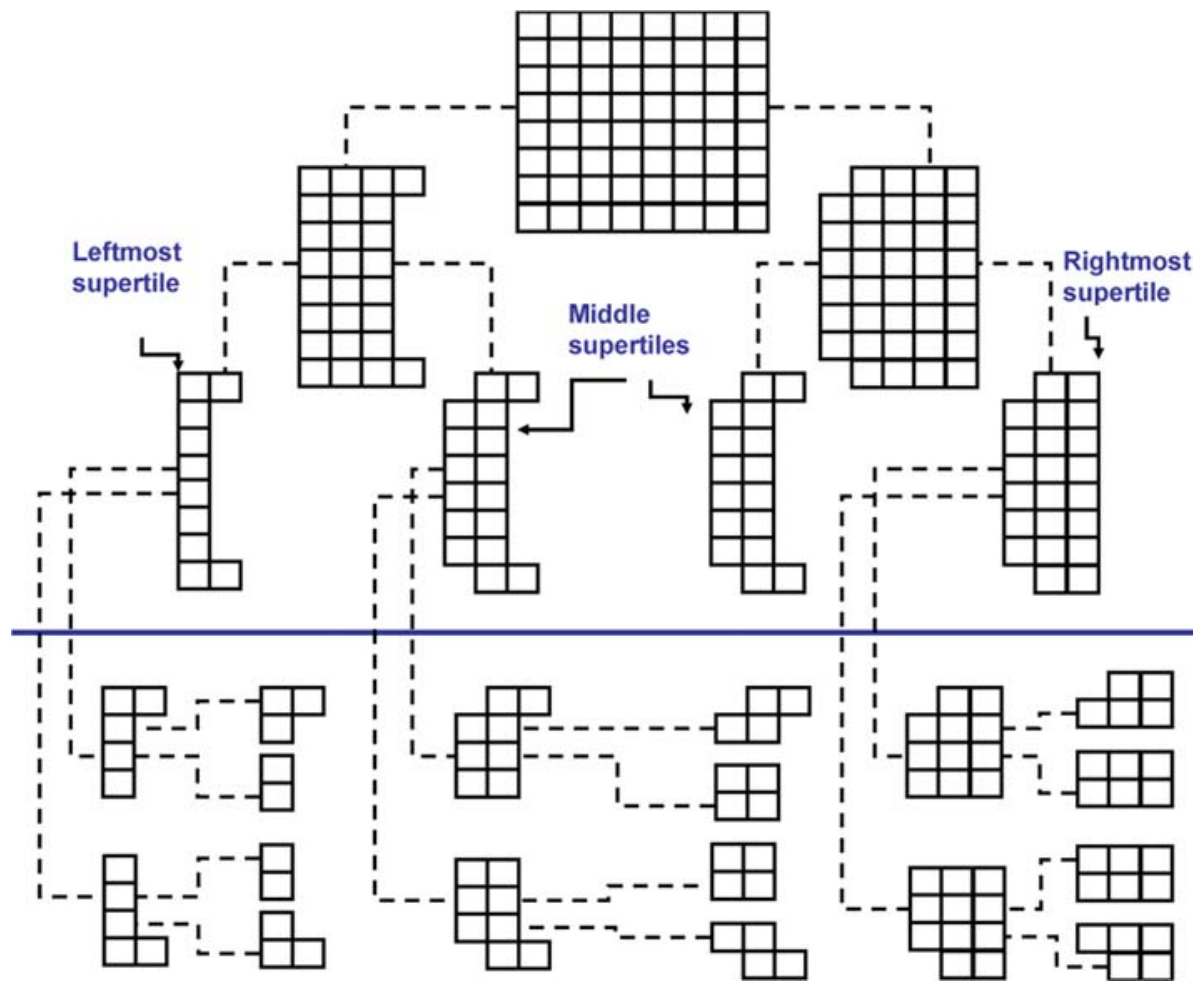

Fig. 5 Decomposition tree for $8 \times 8$ square in the jigsaw technique

Table 2 Algorithm for vertical decomposition. (Horizontal decomposition is symmetric)

Algorithm Decompose Vertically (supertile $S$ ):

- Here $S$ is a supertile with $n$ rows and $m$ columns; $S$ is not necessarily a rectangle.

1. Stop vertical partitioning when width is small enough:

If $m \leq 3$, Decompose Horizontally $(S)$ and return.

2. Find the column along which the supertile is to be partitioned:

Let $i:=\lfloor(m+1) / 2\rfloor$.

Divide supertile $S$ along the $i$ th column into a left supertile $S_{1}$ and right supertile $S_{2}$ such that tiles at position $(1, i)$ and $(n, i)$ belong to $S_{1}$ and the rest of the $i$ th column belongs to $S_{2}$.

3. Now decompose recursively:

Decompose Vertically $\left(S_{1}\right)$

Decompose Vertically $\left(S_{2}\right)$

bins for the even- and odd-columned middle supertiles. For each of the even- or oddcolumned supertiles, each of left and right boundaries of the supertile can have three choices for the glue types. Therefore, there is a constant number of different types of middle supertiles at each level of the decomposition tree. Thus, for vertical decomposition, we need $O(1)$ bins. Each of the supertiles at the end of vertical decomposition undergoes horizontal decomposition. A similar argument applies to the horizontal decomposition as well. Therefore, the number of bins required is $O(1)$. 


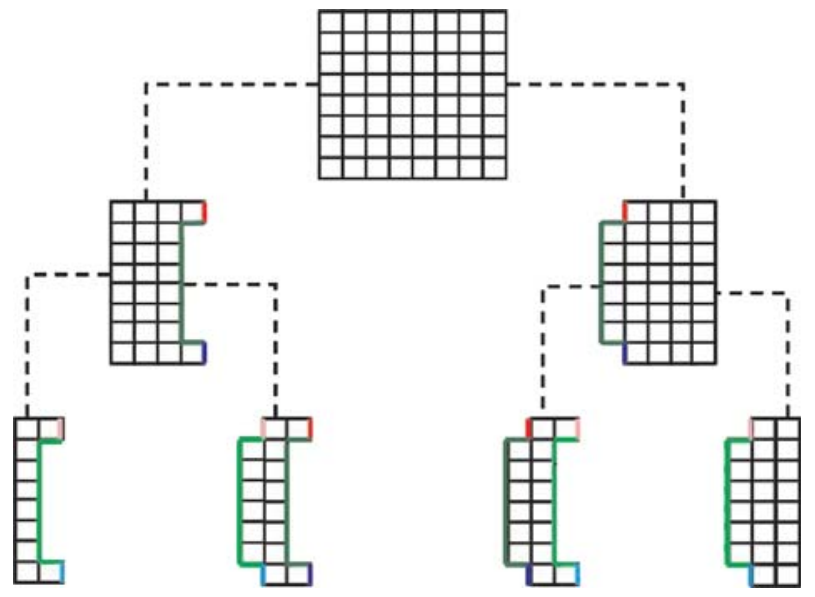

Fig. 6 Assigning glues in the first two vertical decompositions of the jigsaw technique

\subsection{Crazy mixing}

For each stage of a mix graph on $B$ bins, there are up to $\Theta\left(B^{2}\right)$ edges that can be included in the mix graph. By picking which of these edges are included in each stage, $\Theta\left(B^{2}\right)$ bits of information can be encoded into the mix graph per stage. The large amount of information that can be encoded in the mixing pattern of a stage permits a very efficient trade-off between bin complexity and stage complexity. In this section, we consider the complexity of this trade-off in the context of building $n \times n$ squares.

It is possible to view a tile system as a compressed encoding of the shape it assembles. Thus, information theoretic lower bounds for the descriptional or Kolmogorov complexity of the shape assembled can be applied to aspects of the tile system. From this we obtain the following lower bound:

Theorem 3 Any staged assembly system with a fixed temperature and bin complexity $B$ that uniquely assembles an $n \times n$ square with $O(1)$ tile complexity must have stage complexity $\Omega\left(\frac{\log n}{B^{2}}\right)$ for almost all $n$.

Proof The Kolmogorov complexity of an integer $n$ with respect to a universal Turing machine $U$ is $K_{U}(n)=\min |p| s . t U(p)=b_{n}$ where $b_{n}$ is the binary representation of $n$. A straightforward application of the pigeonhole principle yields that $K_{U}(n) \geq\lceil\log n\rceil-\Delta$ for at least $1-\left(\frac{1}{2}\right)^{\Delta}$ of all $n$ (see Li and Vitanyi 1997) for results on Kolmogorov complexity). Thus, for any $\epsilon>0, K_{U}(n) \geq(1-\epsilon) \log n=\Omega(\log n)$ for almost all $n$.

There exists a fixed size Turing machine that takes as input a staged assembly system and outputs the maximum length of the uniquely assembled shape of the system, if there is one. Such a machine that takes as input a system $S=\left\langle M_{r, b},\left\{T_{i, j}\right\}, \tau\right\rangle$ that uniquely assembles an $n \times n$ square will output the integer $n$, and therefore must have size at least $K_{U}(n)$. Therefore, an encoding of $S$ into bits must have size at least $\Omega(\log n)$ for almost all $n$. But, for a constant bounded $\tau$ and $|T|=O(1)$, we can encode $\left\{T_{i, j}\right\}$ and $\tau$ in $O(r b)$ bits and $M_{r, b}$ in $O\left(r b^{2}\right)$ bits for a total $O\left(r b^{2}\right)$ length encoding. Thus, for some constants $c_{1}$ and $c_{2}$ we know that for almost all $n, c_{1} r b^{2} \geq c_{2} \log n$, which yields, $r \geq \frac{c_{2} \log n}{c_{1} b^{2}}$. 
Our upper bound achieves a stage complexity that is within a $O(\log B)$ additive factor of this lower bound:

Theorem 4 For any $n$ and $B$, there is a temperature-2 fully connected staged assembly of an $n \times n$ square using 16 glues, $O(1)$ tiles, $B$ bins, and $O\left(\frac{\log n}{B^{2}}+\log B\right)$ stages.

Proof Within a $O(1)$ additive factor of tile complexity, (Rothemund and Winfree 2000) have reduced the problem of assembling an $n \times n$ square at temperature 2 to the assembly of a length- $\log n$ binary string that uniquely identifies $n$. A straightforward adaptation of the analysis shows that this result also works in the two-handed assembly model used in this paper. Therefore, we focus simply on building an arbitrary $x$-bit input binary string to prove the theorem.

We first show how to build a length- $O\left(B^{2}\right)$ bit string using a temperature-1 system that makes use of $B$ bins, $O(B)$ distinct tiles, and $O(1)$ stages. We then apply a technique similar to that of Theorem 8 to convert this system into a $O(1)$ tile complexity system with an addition of $O(\log B)$ stages. Finally, to get all $x$ bits we can repeat this process $\left\lceil\frac{x}{B^{2}}\right\rceil$ times for a total of $O\left(\frac{x}{B^{2}}+\log B\right)$ stage complexity.

For some arbitrary integer $w$, consider the size $2 w$ tileset and corresponding 3 -stage mix graph given in Fig. 7. For each of $w$ bit positions, there is a corresponding pair of white tiles, one representing the binary value 0 , the other representing 1 . By placing exactly one white tile from each pair into a single bin, a length $w$ bit string is specified. In the transition from stage 1 to stage 2, such a length $w$ string is built for each of $w$ bins, yielding $w$ length $w$ bit strings. These strings can then be concatenated in the transition from stage 2 to 3 to yield a length $w^{2}$ binary string.

For $w=B / 2$, this yields a system with $O(B)$ bins, $O(B)$ tile complexity, and $O(1)$ stage complexity that assembles a length- $B^{2}$ target string. To reduce the tile complexity to $O(1)$, we apply a technique similar to that of Theorem 8 . In particular, we use $O(B)$ bins and $O(\log B)$ stages to create a size $B$ alphabet of macro glues as shown in Fig. 8. Each macro

Tileset:

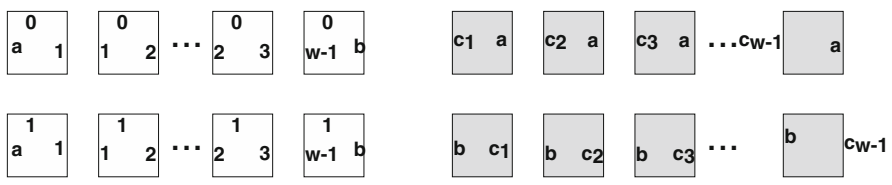

Mix Graph: $\quad$ Example target string: 1001010010100011

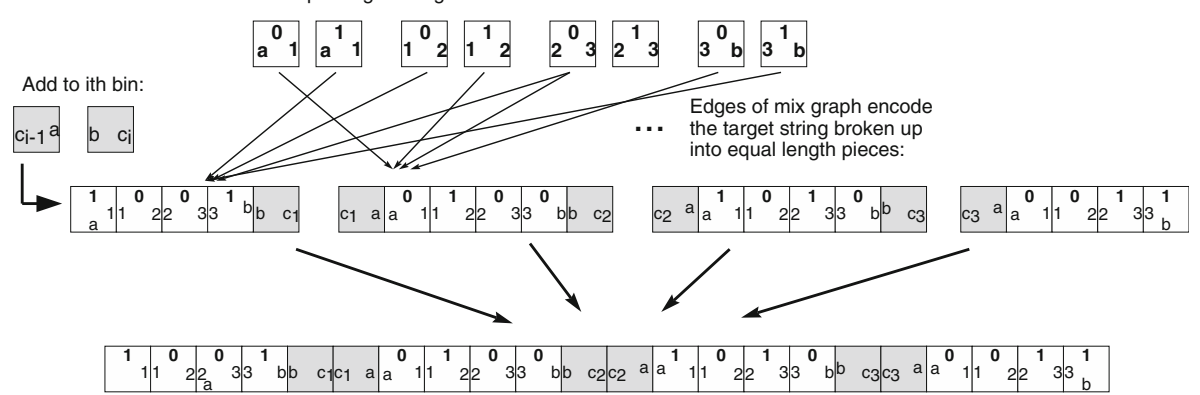

Fig. 7 This tileset and mix graph depict a tile system with $2 w$ tiles and $w$ bins that will assemble an arbitrarily specified length $w^{2}$ binary string 
(a)

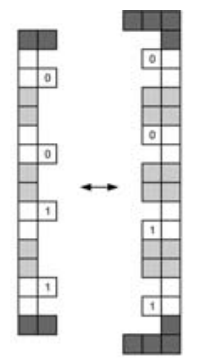

(b)

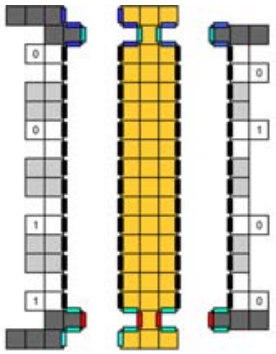

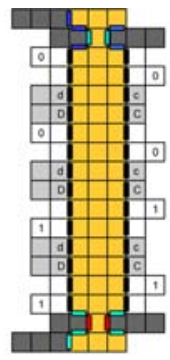

(c)

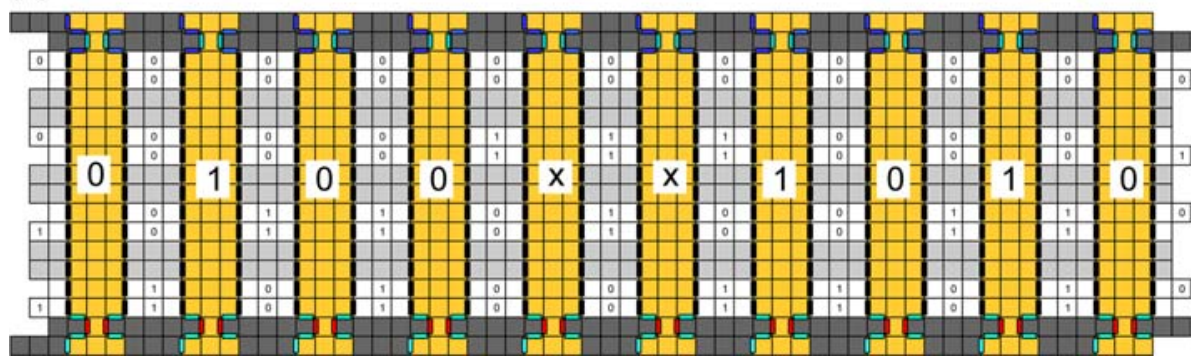

Fig. 8 (a) As described in Sect. 5.3, a collection of supertiles, each in its own bin, can be created such that each supertile encodes a binary string with a sequence of pockets and tabs specified by the binary pattern encoded. These supertiles act as large glues, or macro glues, by combining glue type and geometry to bond only to their exact complement supertile. (b) By combining macro glues onto the surfaces of supertiles, macrotiles can be created whose assembly pattern is the same as that for a corresponding set of singleton tiles. (c) The tile set of from Fig. 7 can be simulated with macro tiles to create a binary string using only $O(1)$ tile complexity. The example assembly shown here corresponds to the middle section of the example assembly from Fig. 7

glue is a supertile that consists of a string of tiles representing bits of a binary string. Further, with the same bin and stage complexity we create a parallel set of complement macro glues as shown in Fig. 8(a). Note that when combined into the same bin, two macro glues will only attach to one another if they are exact complements (have the same binary encoding). By design, the tooth like geometry of the macro glues provides that even a single bit difference between two macro glues excludes even a single bond from attaching.

Given this set of macro glues, we now conceptually index the set of distinct glues from the size $O(B)$ tileset of Fig. 7 and assign each glue a corresponding macro glue whose binary string matches the index of the glue. Next, we attach macro glues to the long thin supertiles shown in Fig. 8 which can be created using a slightly modified version of the line algorithm from Sect. 3. In particular, for each element of the tileset from Fig. 7, we attach macro glues corresponding to the east and west glues of the singleton tile. Further, we can assign a glue representing ' 0 ', ' 1 ', or 'nothing' on the north surface of each macro tile, according to which glue the corresponding singleton tile displays on its north side.

Once we have built this set of macro tiles, we mix them according to the same mixing algorithm for the size $O(B)$ tile set, but instead replace each singleton tile with its corresponding macro tile. By design, the macro glues attach exactly as the basic glues they are built to emulate. The result is thus a length- $w^{2}$ binary string encoded on the north surface of the assembled macro tiles. 
Finally, to get a length- $x$ string, we can repeat this process $\left\lceil\frac{x}{B^{2}}\right\rceil$ times for a total of $O\left(\frac{x}{B^{2}}+\log B\right)$ stage complexity. Given this string, the technique of (Rothemund and Winfree 2000) is easily adapted to take into account the $\log B$ vertical magnification factor we introduce by utilizing the macro glue construction. Further, while the technique of (Rothemund and Winfree 2000) is temperature 2 rather than 1, this is not a problem as we can simply double the strength of each glue in our construction to make it a temperature 2 system. Details of applying the square building set from (Rothemund and Winfree 2000) are straightforward and applications of the technique to similar problems are considered in (Aggarwal 2005; Kao and Schweller 2006).

Finally, we observe that the construction used here can be designed to achieve full connectivity and is planar. Further, the construction of (Rothemund and Winfree 2000) maintains this full connectivity and planarity, yielding the result.

We conjecture that this stage complexity bound can be achieved by a temperature-1 assembly by judicious use of the jigsaw technique.

\section{Assembly of general shapes}

In this section, we describe a variety of techniques for manufacturing arbitrary shapes using staged assembly with $O(1)$ glues and tiles.

\subsection{Spanning-tree technique}

The spanning-tree technique is a general tool for making an arbitrary shape with the connectivity of a tree. We start with a sequential version of the assembly:

Theorem 5 Any shape $S$ with $n$ tiles has a partially connected temperature-1 staged assembly using 2 glues, at most 16 tiles, $O(\log n)$ bins, and $O(\operatorname{diameter}(S))$ stages.

Proof Take a breadth-first spanning tree of the adjacency graph of the shape $S$. The depth of this tree is $O(\operatorname{diameter}(S))$. Root the tree at an arbitrary leaf. Thus, each vertex in the tree has at most three children. Color the vertices with two colors, black and white, alternating per level. For each edge between a white parent and a black child, we assign a white glue to the corresponding tiles' shared edge. For each edge between a black parent and a white child, we assign a black glue to the corresponding tiles' shared edge. All other tile edges receive the null glue. Now a tile has at most three edges of its color connecting to its children, and at most one edge of the opposite color connecting to its parent.

To obtain the sequential assembly, we perform a particular postorder traversal of the tree: at node $v$, visit its child subtrees in decreasing order of size. To combine at node $v$, we mix the recursively computed bins for the child subtrees together with the tile corresponding to node $v$. The bichromatic labeling ensures unique assemblage. The number of intermediate products we need to store is $O(\log n)$, because when we recurse into a second child, its subtree must have size at most $2 / 3$ of the parent's subtree.

Figure 9 illustrates spanning tree method for assembling $3 \times 3$ square. In general, this construction is nonplanar: the trees may fit together like a key in a keyhole.

The stage complexity of the spanning-tree technique can be reduced by parallelization, at the cost of more bins: 

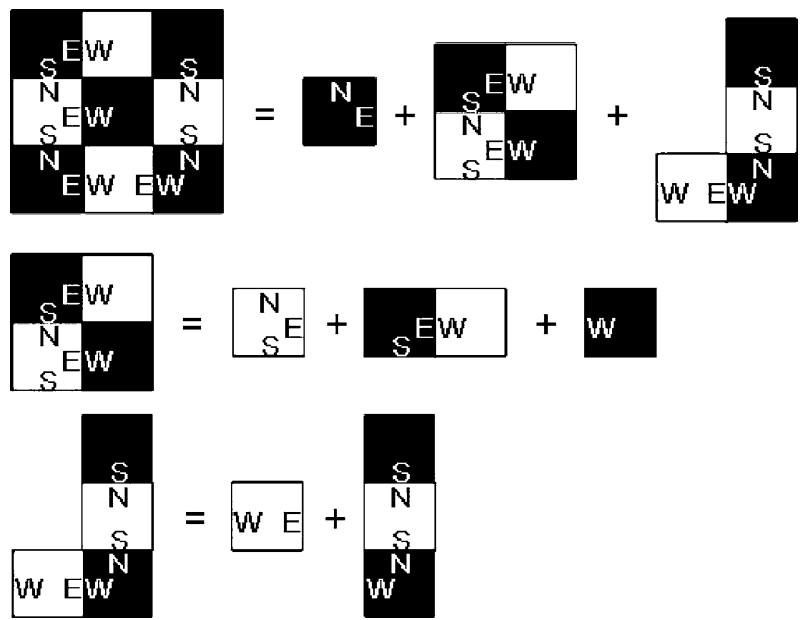

Fig. 9 Spanning-tree method for assembling a $3 \times 3$ square

Theorem 6 Any shape $S$ with $n$ tiles has a partially connected temperature-1 staged assembly using $O(1)$ tiles, $O(\log n)$ stages, and $O(n / \log n)$ bins.

Proof As before, we consider a two-colored breadth-first spanning tree. To build an $n$-tile tree, split this tree into two trees of at most $2 n / 3$ nodes. Recursively build these two trees, and then mix the two resulting bins of supertiles together. If we continue this recursion down to individual nodes (tiles), we get $n$ such trees and the stage complexity reduces to $O(\log n)$, but the bin complexity is now $O(n)$. We can do better if we recurse until we get $n / \log n$ trees of size $\log n$ each. By Theorem 5, each of these trees can be built using $O(\log n)$ stages and $O(1)$ bins. Thus we need $n / \log n$ bins in total. These trees can be combined using another $O(\log n)$ stages to get the $n$-tile tree.

\subsection{Scale factor 2}

Although the spanning-tree technique is general, it probably manufactures structurally unsound assemblies. Next we show how to obtain full connectivity of general shapes, while still using only a constant number of glues and tiles.

Theorem 7 Any simply connected shape has a staged assembly using a scale factor of 2, 8 glues, $O(1)$ tiles, $O(n)$ stages, and $O(n)$ bins. The construction maintains full connectivity.

Proof Slice the target shape with horizontal lines to divide the shape into $1 \times k$ strips for various values of $k$, which scale to $2 \times 2 k$ strips (uniform factor- 2 scaling of the target shape). These strips can be adjacent along horizontal edges but not along vertical edges. Define the strip graph to have a vertex for each strip and an edge between two strips that are adjacent along a horizontal edge. Because the shape is simply connected (hole-free), the strip graph is a tree. Root this tree at an arbitrary strip, defining a parent relation.

A recursive algorithm builds the subtree of the strip graph rooted at an arbitrary strip $s$. As shown in Fig. 10(a), the strip $s$ may attach to the rest of the shape at zero or more places on its top or bottom edge. One of these connections corresponds to the parent of $s$ (unless $s$ 
(a)

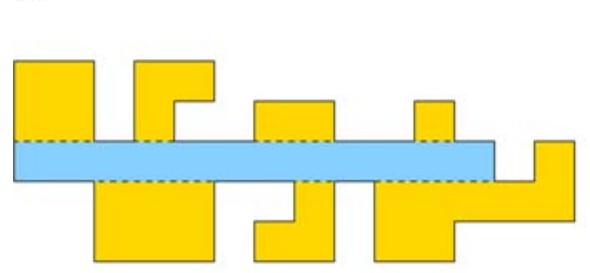

(b)
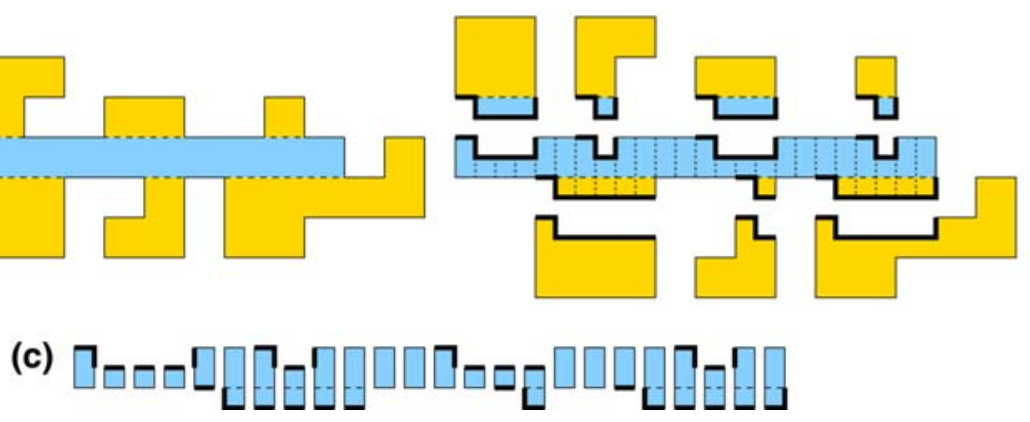

Fig. 10 Constructing a horizontal strip in a factor-2 scaled shape (a), augmented by jigsaw tabs and pockets to attach to adjacent pieces (b), proceeding column-by-column (c)

is the overall root). As shown in Fig. 10(b), our goal is to form each of these attachments using a jigsaw tab/pocket combination, where bottom edges have tabs and top edges have pockets, extending from the rightmost square up to but not including the leftmost square. Factor-2 scaling ensures that it is always possible to create these tabs and pockets.

The horizontal edges of each tab or pocket uses a pair of glues. The unit-length upper horizontal edge uses one glue, and the possibly longer lower horizontal edge uses the other glue. The pockets at the top of strip $s$ use a different glue pair from the tabs at the bottom of strip $s$. Furthermore, the pocket or tab connecting $s$ to its parent uses a different glue pair from all other pockets and tabs. Thus, there are four different glue pairs (for a total of eight glues). If the depth of $s$ in the rooted tree of the strip graph is even, then we use the first glue pair for the top pockets, the second glue pair for the bottom tabs, except for the connection to the parent which uses either the third or fourth glue pair depending on whether the connection is a top pocket or a bottom tab. If the depth of $s$ is odd, then we reverse the roles of the first two glue pairs with the last two glue pairs. All vertical edges of tabs and pockets use the same glue, 8 .

To construct the strip $s$ augmented by tabs and pockets, we proceed sequentially from left to right, as shown in Fig. 10(c). The construction uses two bins. At the $k$ th step, the primary bin contains the first $k-1$ columns of the augmented strip. In the secondary bin, we construct the $k$ th column by brute force in one stage using 1-3 tiles and 0-2 distinct internal glues plus the desired glues on the boundary. Because the column specifies only two glues for horizontal edges, at the top and bottom, we can use any two other glues for the internal glues. All of the vertical edges of the column use different glues. If $k$ is odd, the left edges use glues 1-3 and the right edges uses glues 4-6, according to $y$ coordinate; if $k$ is even, the roles are reversed. (In particular, these glues do not conflict with glue 8 in the tabs and pockets.) The only exception is the first and last columns, which have no glues on their left and right sides, respectively. Now we can add the secondary bin to the primary bin, and the $k$ th column will uniquely attach to the right side of the first $k-1$ columns. In the end, we obtain the augmented strip.

During the building of the strip, we attach children subtrees. Specifically, once we assemble the rightmost column of an attachment to one or two children strips, we recursively assemble those one or two children subtrees in separate bins, and then mix them into $s$ 's primary bin. Because the glues on the top and bottom sides of $s$ differ, as do the glues of $s$ 's parent, and because of the jigsaw approach, each child we add has a unique place to 
attach. Therefore we uniquely assemble $s$ 's subtree. Applying this construction to the root of the tree, we obtain a unique assembly of the entire shape.

\subsection{Simulation of one-stage assembly with logarithmic scale factor}

In this section, we show how to use a small number of stages to combine a constant number of tile types into a collection of supertiles that can simulate the assembly of an arbitrary set of tiles at temperature $\tau=1$, given that these tiles only assemble fully connected shapes.

Theorem 8 Consider an arbitrary single stage, single bin tile system with tile set $T$, all glues of strength at most 1 , and that assembles a class of fully connected shapes. There is a temperature-1 staged assembly system that simulates the one-stage assembly of $T$ up to an $O(\log |T|)$ size scale factor using 3 glues, $O(1)$ tiles, $O(|T|)$ bins, and $O(\log \log |T|)$ stages. At the cost of increasing temperature to $\tau=2$, the construction achieves full connectivity.

Proof Suppose the $T$ uses $c$ distinct glue types. As described in Fig. 11, the initial stage of assembly can use three distinct tile types that assemble into a supertile representing 0 in a first bin, and three tile types for the assembly of a supertile representing 1 in a second bin. We can then split these supertiles into four groups and attach tile types $a$ and $A$ as shown in Fig. 11. The third stage mixes all possible combinations of supertiles attached to tile type $a$ with those attached with type $A$ to get a distinct supertile for each possible 4-bit binary string. This process can be repeated to obtain all possible length- 8 bit strings, and so on. Thus, within $O(\log \log c)$ stages we can obtain at least $c$ distinct binary strings of length at most $O(\log c)$.

Repeating this process four times produces an alphabet of glue types for each tile side. As shown in Fig. 11, we can make the geometry of identical bits for opposite directions (north/south, east/west) be interlocking. Thus, when two glues are lined up against each

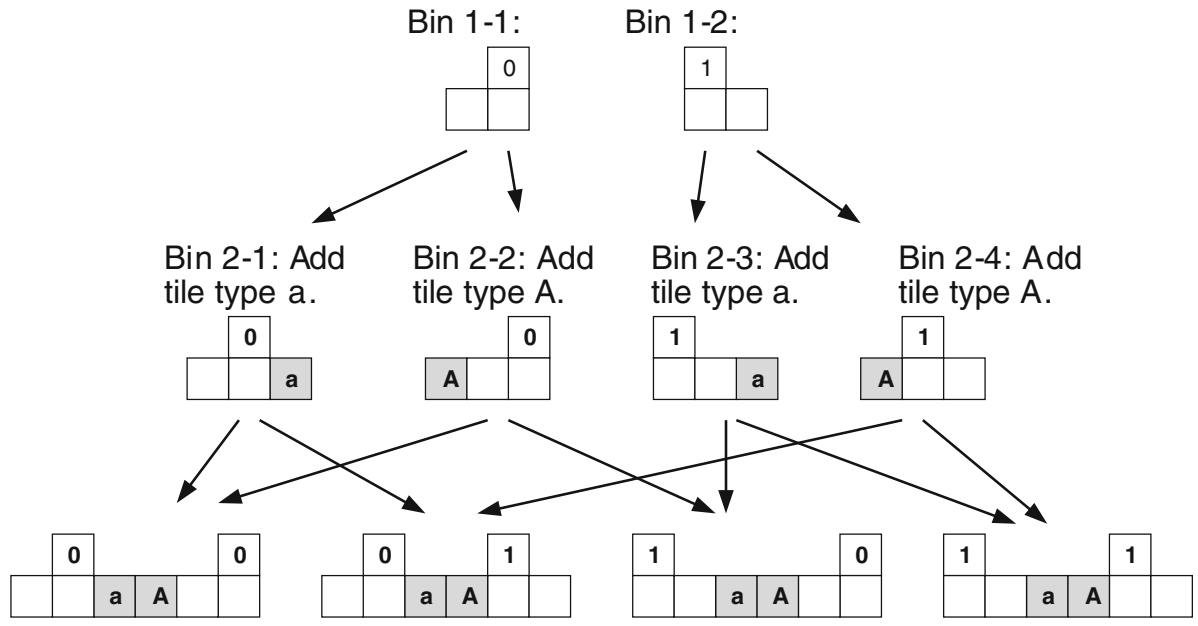

Bin 1-2:

Fig. 11 (a) Using $O(1)$ tile types and $O(\log r)$ stages, we can assemble $2^{r}$ different supertiles, each encoding a distinct $r$ bit binary string. (b) By creating two versions of each string and appending tiles to the ends we can enforce that identical strings combine while distinct strings do not. Note that even if a single bit differs between two strings, the rigid geometry of the supertiles ensure that no tiles will be able to bond 
other, if all bits match, the two supertiles can lock together and get a full bonding. However, due to the interlocking geometry, if even a single bit does not match, this mismatch will prevent the two supertiles from getting close enough to get even a single bond. Further, to prevent shifting of strings that share prefixes/suffixes, we can attach the interlocking dark tiles shown in Fig. 11(b).

Finally, given the four alphabets of glues with each glue type in a separate bin, we can bring together arbitrary combinations of four to create macro tiles as shown in Fig. 12. We can thus create a set of macro tiles that will bond in the same fashion as any given target $\tau=1$ tile system. The holes in the constructed shape can trivially be filled in in a nonplanar fashion by adding in a constant size set of filler tiles.

Note that the construction does not work for simulating $\tau=2$ systems if we restrict ourselves to a constant bounded temperature. This is because a single glue match for a macro tile yields a large, nonconstant number of bonds. Further, note that when a macro tile attaches at a position adjacent to two or more already attached macro tiles, it cannot attach within the plane, making the construction inherently nonplanar.

Extending this simulation to temperature-2 one-stage systems is an open problem.

\subsection{Assembly of monotone shapes}

Theorem 9 Any monotone shape has a fully connected temperature-1 staged assembly using 9 glues, $O(1)$ tiles, $O(\log n)$ stages, and $O(n)$ bins, where $n$ is the side length of the smallest square bounding $S$.

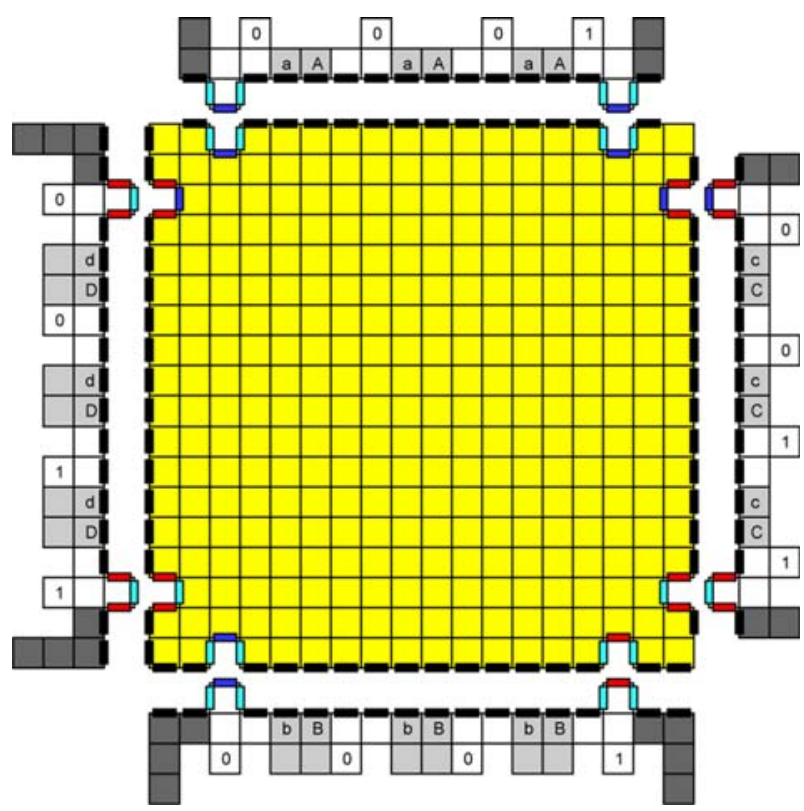

Fig. 12 By constructing an alphabet of binary strings for each of the four possible tile sides, arbitrary combinations of four can be brought together to assemble macro tiles. This permits the simulation of $\tau=1$ tile systems with macro blocks using only $O(1)$ tile types 
Proof We assume wlog that the shape is $\mathrm{x}$-monotone, which means its intersection with any vertical line is connected. We use the similar construction that we used for building square. We first decompose the shape horizontally to get long thin supertiles which we already know how to build. Here we will only discuss horizontal decomposition. During horizontal decomposition, the challenge is to decompose a supertile $S$ into a left and a right supertile that can be combined uniquely. We decompose $S$ horizontally only when the number of columns in $S$ is greater than 3, otherwise, we just need vertical decomposition. Let $i, i+1$, and $i+2$ be the three columns roughly in the middle of the supertile $S$. Column $i$ is adjacent to the column $i+1$ at certain locations. Since the shape is x-monotone, the tiles in column $i$ adjacent to column $i+1$ form a connected component. Same is the case with tiles in column $i+2$ that are adjacent to column $i+1$.

If the number of adjacent tiles between column $i$ and $i+1$ is $\leq 3$, we simply cut $S$ between column $i$ and $i+1$. Otherwise if the number of tiles in column $i+2$ adjacent to the tiles in column $i+1$ is $\leq 3$, we can break $S$ between columns $i+1$ and $i+2$. See Fig. 13 (top).

If column $i+1$ is adjacent to both columns in more than three tiles, we find the tiles in column $i+1$ that are adjacent to both columns. These tiles form a connected component due to monotonicity. If the number of such tiles $\geq 3$ we can create a jigsaw tab/pocket combination at column $i+1$. See Fig. 13 (middle). Notice the left supertile is not monotone anymore because of the last column. But we can ignore the last column because it will never be one of the three middle columns until the supertile contains only three columns and at that point we don't need horizontal decomposition any more.

If number of tiles in column $i+1$ that are adjacent to both columns is $<3$, we decompose $S$ by creating an elbow see Fig. 13 (below). To create an elbow: assume without loss of generality that the highest tile in column $i$ adjacent to column $i+1$ is lower than the highest tile in column $i+2$ adjacent to column $i+1$. We cut the column $i+1$ such that the tiles in the column that are either adjacent to column $i$ or below any such tile belong to the left supertile and the rest of the column belong to right supertile.

The horizontal decomposition uses only constant number of only 9 glues thus $O(1)$ tiles. The decomposition tree is balanced so we need only $O(\log n)$ stages. The number

Fig. 13 Assembling a monotone shape
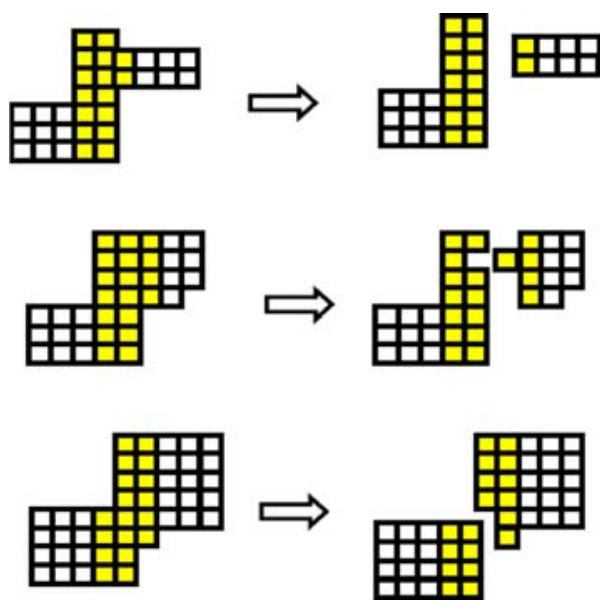
of bins required can be $O(n)$ because we may need to keep each column in a separate bin.

\section{Fast counters at temperature $\tau=1$}

One of the most powerful and prevalent tools in the algorithmic self-assembly literature is the counter (Rothemund and Winfree 2000; Adleman et al. 2001; Aggarwal 2005; Kao and Schweller 2006; Winfree 1998; Barish et al. 2005). A set of tiles that implement a counter are tiles that assemble into a pattern such that successive positive integer positions are encoded into successive positions in the assembled shape. Such constructions will typically then control the length of the assembled shape by stopping growth when the counter reaches its maximum value. In this section we introduce a new method of building counters in the tile assembly model that takes advantage of the power of staged assembly. We argue that our approach yields some important benefits in terms of assembly speed and temperature $\tau=1$. Given the proven utility of counter assemblies, we provide our construction as a primitive tool that may be useful in the development of more efficient assembly systems.

The most typical example of a counter consists of a tile set where each tile type is conceptually assigned either a ' 0 ' or a ' 1 ' binary label. For some specified value $k$, such a system assembles a $k \times 2^{k}$ rectangle such that for any row $i$ in the assembly, the $k$ tiles in the row $i$ encode the binary value of $i$ by their assigned labels.

Counters under the standard single stage model suffer from two drawbacks. First, they require temperature $\tau=2$ to work. Second, all the constructions to date in the literature are designed so that the $i^{\text {th }}$ value of the counter cannot attach/assemble until the 1 st through $i-1$ values have already assembled. This creates a lower bound of $\Omega(n)$ assembly time for these constructions (see (Adleman et al. 2001) for a definition of assembly time under the standard model).

In our construction of a binary counter, we attempt to improve upon both of these drawbacks. First, our construction utilizes temperature $\tau=1$. Second, the construction may assembly in a parallel manner. That is, the supertile encoding the value $i$ can attach to the supertile encoding the value $i+1$ at any time, regardless of whether or not the supertile representing the value $i-1$ has attached to anything. While a definition of assembly time under the two-handed assembly model has not yet been developed, it is plausible that this parallelism could yield a substantial reduction in assembly time for a reasonable model.

\subsection{Counter construction}

To implement the staged assembly binary counter, we design a mixing algorithm to yield two batches of supertiles as shown in Fig. 14, each including a list of long thin supertiles encoding a bit pattern of interlocking teeth on the north and south surface of the supertile in the same fashion as Theorem 8. In particular, the first batch will consist of supertiles whose pattern of interlocking teeth on the north face of the supertile encode the binary string obtained by incrementing the binary string encoded on the south face of the supertile by one. The second batch is similar, but the string encoded on the north and south face of each supertile is not incremented.

By design, the glues on the north and south faces of each supertile in either batch are distinct, making attachment among supertiles impossible. However, we can make the north glues used in the first batch the same as the south glues used in the second batch, and vice 
(a)
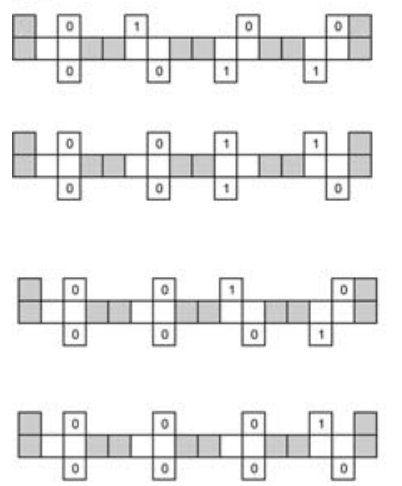

(b)
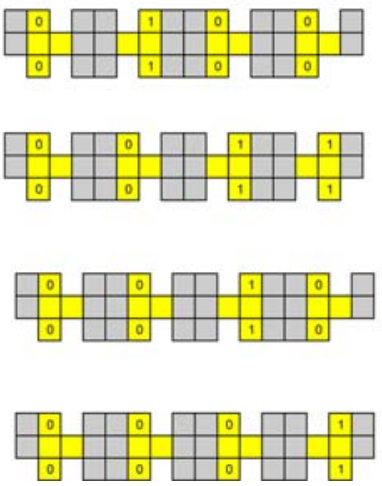

(c)

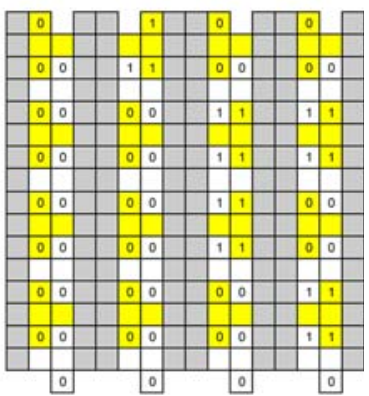

Fig. 14 (a and b) With $O(1)$ tile complexity, $O(1)$ bin complexity, and $O(k)$ stage complexity, two separate batches of supertiles can be created, each containing $2^{2^{k}}$ distinct supertiles. (c) When combined, supertiles may attach together by alternating between supertiles from each group. Further, attachment is only possible between supertiles whose binary strings denote values that are of difference exactly one. The effect is thus an assembly whose bit pattern encoded row by row represents a counter incrementing by one until the maximum value is reached, yielding a length $O\left(2^{2^{k}}\right)$ assembly. In this example of length 4 strings, only 4 of the possible 16 supertiles are shown. With this construction, in contrast to single stage assemblies, two successive counter values may attach independent of whether or not previous values have attached. Thus, the resultant structure should assemble much quicker than other methods in which each row of a counter must be added in succession, starting from an initial seed row

versa. From this we get that when the two batches are mixed together supertiles may assemble by alternating between supertiles from the first batch and supertiles from the second batch.

Further, due to the geometry encoded on the surface of each supertile, each supertile attaches above a supertile whose binary value is exactly one less than its own. Thus, any assembled structure consists of a chain of rows, each row representing an incremented binary value. Therefore the unique terminal assembly is such that the northmost face is a supertile encoding the highest value string of all 1's, while the south face consists of the supertile representing the string of all 0's.

To see how to assemble the binary strings used in this construction, consider the problem of assembling a set of supertiles such that each of the $2^{2^{i}}$ length $2^{i}$ binary strings is represented by a supertile encoding the string on its south surface. Further, for each such supertile in the set, require that it encodes the value encoded on its south surface incremented by 1 on its north surface (assume the all 1's string incremented is the all 0's string). Denote this set as $X_{i}$. Such a set is essentially the first batch of Fig. 14, and a straightforward modification of the following technique can yield the second batch as well.

Now, to obtain a bin whose unique assemblies are $X_{i}$, it is sufficient to obtain 2 bins whose assemblies union are equal to $X_{i}$, as these bins can be combined within 1 stage. Let $I_{i}$ ( $I$ for incremented strings) denote the subset of strings in $X_{i}$ minus the string whose south surface is all 1's. Let $R_{i}$ ( $R$ for rollover strings) simply be the supertile encoding all 1 's on the south face and all 0's on the north face. Finally, define a third set $S_{i}$ not contained in $X_{i}$, where $S_{i}$ is the set of all length $i$ strings encoding the same value on the south and north face of the supertile.

To describe how to attain a bin with the set $X_{i}$ as uniquely produced supertiles, we show how to recursively compute the three sets $S_{i}, I_{i}$, and $R_{i}$. Assume, as depicted in Fig. 15, that 


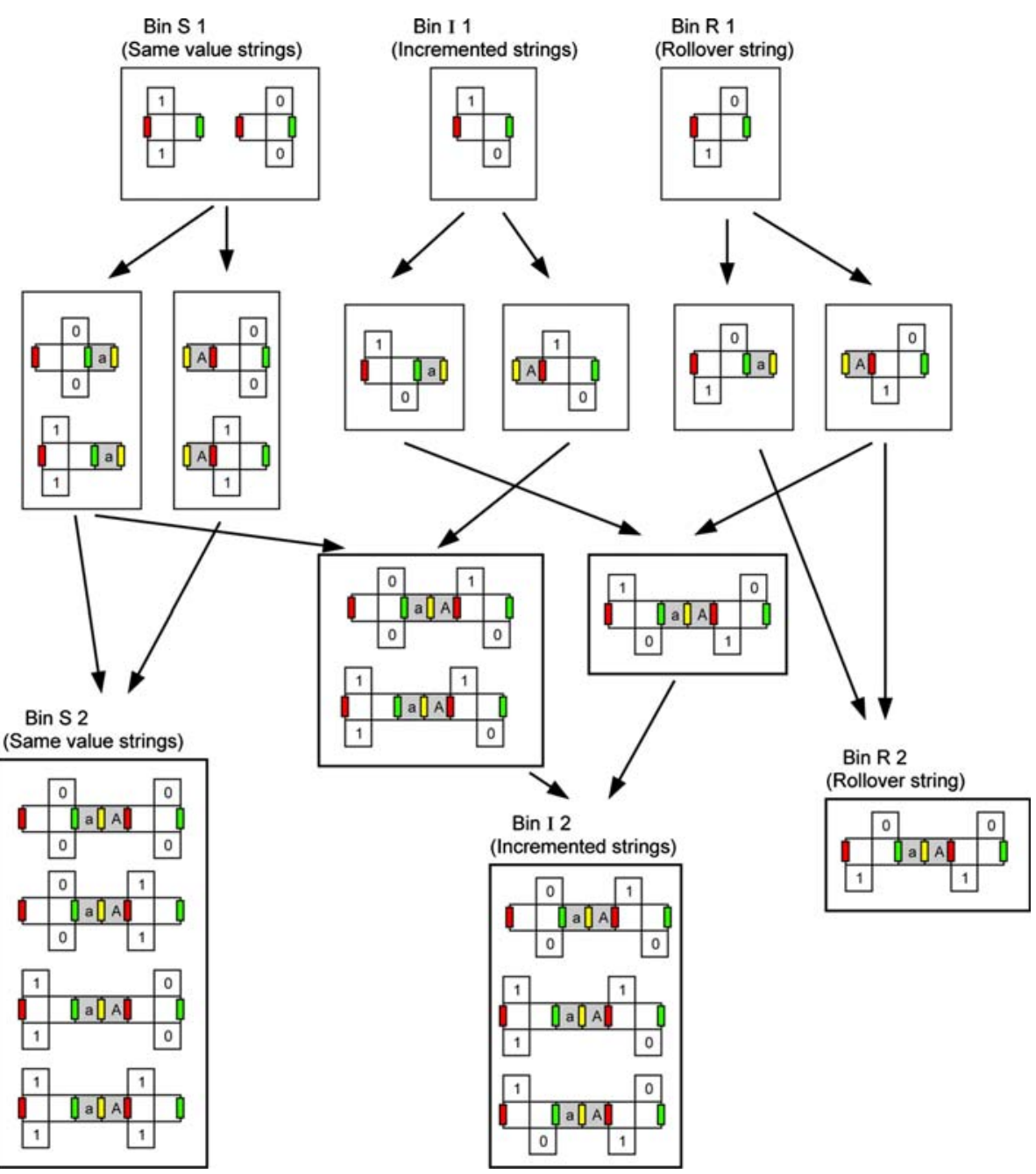

Fig. 15 Supertiles capable of binary counting can be constructed efficiently by a simple recursive mixing algorithm. A set of binary strings of length $x$ can be assembled in $O(1)$ bin complexity and $O(\log x)$ stage complexity

each supertile in the sets $S, I$, and $R$ must have a strength 1 red and green glue on the west most and east most center edge respectively.

Recursively, assume we already have 3 separate bins containing $S_{i / 2}, I_{i / 2}$, and $R_{i / 2}$. Within a single stage, split the contents of each of these three bins into 2 separate bins (for a total of six distinct bins). Denote the bins by $S_{i / 2}^{a}$ and $S_{i / 2}^{A}$ etc. For the $a$ bins, add tile $a$ from Fig. 15. For the $A$ bins, add tile $A$.

Now combine sets $S_{i / 2}^{a}$ and $S_{i / 2}^{A}$. This yields a bin containing the set of all length $i$ binary strings that have the same values on the north and south faces, which is the set $S_{i}$.

Now combine set $S_{i / 2}^{a}$ and $I_{i / 2}^{A}$. This yields a set of supertiles that is a subset of $I_{i}$, namely the strings (encoded on the south face of the supertile) whose least significant 0 occurs in the right half of the string. The remaining set of $I_{i}$, the strings whose least significant 0 
occurs in the left half of the string, is obtained by combining $I_{i / 2}^{a}$ and $R_{i / 2}^{A}$. A third stage thus yields the set $I_{i}$.

Finally, the set $R_{i}$ is obtained by combining $R_{i / 2}^{a}$ and $R_{i / 2}^{A}$.

As base case for this recursive mixing procedure, we can build the sets for $i=1$ by brute force with distinct tile types. This technique uses at most 6 bins and 3 stages per recursion level. Thus, the desired set $X_{x}$ can be obtained in $O(1)$ bins and $O(\log x)$ stages. The procedure for extending size 1 strings to size 2 strings is depicted in Fig. 15.

\subsection{Counting up to general $n$}

The counter described in Sect. 6.1 counts from value 0 up to $2^{2^{k}}-1$ for a specified value $k$ using $k$ stages, $O(1)$ bins, and $O(1)$ tile complexity. However, this construction clearly is not immediately capable of assembling supertiles of arbitrary length $n$. In contrast, constructions exist at $\tau=2$ under the single stage model such that the exact length of counters can be specified. This can typically be done by specifying an initial first value of the counter as these systems always start from a seed value. However, with our approach this is much more difficult.

Currently, we have a complex construction combining the technique of Theorem 4 with the binary counter system of Sect. 6.1 to yield unique assembly of a counter of any length $n$ at temperature $\tau=1, O(B)$ bin complexity, and $O\left(\frac{\log n}{B^{2}}+\log B\right)$ stage complexity. However, we do not include the details of this construction as it is very complex and as of yet does not have direct application to building shapes of interest, such as squares. However, we conjecture that this technique can yield a square building scheme that improves Theorem 4 to a $\tau=1$ construction.

\section{Future directions}

There are several open research questions stemming from this work.

One direction is to relax the assumption that, at each stage, all supertiles self-assemble to completion. In practice, it is likely that at least some tiles will fail to reach their terminal assembly before the start of the next stage. Can a staged assembly be robust against such errors, or at least detect these errors by some filtering, or can we bound the error propagation in some probabilistic model?

Another direction is to develop a model of the assembly time required by a mixing operation involving two bins of tiles. Such models exist for (one-stage) seeded selfassembly — which starts with a seed tile and places singleton tiles one at a time-but this model fails to capture the more parallel nature of two-handed assembly in which large supertiles can bond together without a seed.

Another interesting direction would be to consider nondeterministic assembly in which a tile system is capable of building a large class of distinct shapes. Is it possible to design the system so that certain shapes are assembled with high probability?

Another research direction is the consideration of 3D assembly. We have focused on twodimensional constructions in this paper which provides a more direct comparison with previous models, and is also a case of practical interest, e.g., for manufacturing sieves. Many of our results, in theory, also generalize to 3D (or any constant dimension), at the cost of increasing the number of glues and tiles. For example, the spanning-tree model generalizes trivially, and a modification to the jigsaw idea enables many of the other results to carry over. However, 3D assembly in practice is much harder than 2D assembly, stemming in part from 
the fact that $2 \mathrm{D}$ assembly systems in practice make use of 3 dimensions. How to properly model and address the difficulties of 3D assembly is an important research direction. In particular, combining our staged assembly techniques with existing error correcting mechanisms seems a potentially fruitful direction for further research.

Finally, experimental validation of our model and techniques is an extremely important direction for future work. It is likely that simulations and implementations of staged assembly techniques will yield key insights into the model, providing a road map for future work.

Acknowledgments We thank M. S. AtKisson and Edward Goldberg for extensive discussions about the bioengineering application. E. D. Demaine and M. L. Demaine research was partially supported by NSF CAREER award CCF-0347776 and DOE grant DE-FG02-04ER25647. M. Ishaque, D. L. Souvaine and E. Rafalin research was partially supported by NSF grant CCF-0431027. E. Rafalin's work was performed at Tufts University.

\section{References}

Adleman LM (2000) Toward a mathematical theory of self-assembly. Technical Report 00-722, Department of Computer Science, University of Southern California

Adleman L, Cheng Q, Goel A, Huang M-D (2001) Running time and program size for self-assembled squares. In: Proceedings of the 33rd annual ACM symposium on Theory of Computing, pp 740-748

Adleman L, Cheng Q, Goel A, Huang M-D, Kempe D, de Espanés PM, Rothemund PWK (2002) Combinatorial optimization problems in self-assembly. In: Proceedings of the thirty-fourth annual ACM symposium on Theory of Computing, pp 23-32 (electronic), New York, ACM

Aggarwal G, Cheng Q, Goldwasser MH, Kao M-Y, de Espanes PM, Schweller RT (2005) Complexities for generalized models of self-assembly. SIAM J Comput 34(6):1493-1515

Barish RD, Rothemund PWK, Winfree E (2005) Two computational primitives for algorithmic selfassembly: Copying and counting. Nano Lett 5(12):2586-2592

Kao M-Y, Schweller R (2006) Reducing tile complexity for self-assembly through temperature programming. In: Proceedings of the 17th annual ACM-SIAM symposium on discrete algorithm, pp 571-580

Li M, Vitanyi P (1997) An introduction to komogorov complexity and its applications, 2nd edn. Springer Verlag, New York

Mao C, LaBean TH, Reif JH, Seeman NC (2000) Logical computation using algorithmic self-assembly of DNA triple-crossover molecules. Nature 407:493-496

Park SH, Pistol C, Ahn SJ, Reif JH, Lebeck AR, Dwyer C, LaBean TH (2006) Finite-size, fully addressable DNA tile lattices formed by hierarchical assembly procedures. Angewandte Chemie 45:735-739

Reif J (1999) Local parallel biomolecular computation. In: Proceedings of DNA-based computers, pp 217254

Rothemund PWK (2006) Folding DNA to create nanoscale shapes and patterns. Nature 440:297-302

Rothemund PWK, Winfree E (2000) The program-size complexity of self-assembled squares. In: Proceedings of the 32nd annual ACM symposium on Theory of Computing, pp 459-468

Rothemund PWK, Papadakis N, Winfree E (2004) Algorithmic self-assembly of DNA sierpinski triangles. PLoS Biol 2(12):e424

Seeman NC (1998) DNA nanotechnology. In: Siegel RW, Hu E, Roco MC (eds) WTEC workshop report on R\&D status and trends in nanoparticles, nanostructured materials, and nanodevices in the United States

Shih WM, Quispe JD, Joyce GF (2004) A 1.7-kilobase single-stranded DNA that folds into a nanoscale octahedron. Nature 427:618-621

Soloveichik D, Winfree E (2004) Complexity of self-assembled shapes. In: Revised selected papers from the 10th international workshop on DNA computing. Lecture notes in computer science, vol 3384. Milan, Italy, pp 344-354

Somei K, Kaneda S, Fujii T, Murata S (2006) A microfluidic device for DNA tile self-assembly. In: DNA computing. Springer, Berlin/Heidelberg, pp 325-335

Wang H (1961) Proving theorems by pattern recognition-II. Bell System Tech J 40(1):1-41

Winfree E (1998) Algorithmic self-assembly of DNA. PhD thesis, California Institute of Technology, Pasadena

Winfree E, Liu F, Wenzler LA, Seeman NC (1998) Design and self-assembly of two-dimensional DNA crystals. Nature 394:539-544 